\title{
The prognostic value of a seven-IncRNA signature in patients with esophageal squamous cell carcinoma: a IncRNA expression analysis
}

Nuo-Qing Weng ${ }^{1,2+}$, Jun Chi ${ }^{1,2,3 \dagger}$, Jing Wen ${ }^{1,2,4 \dagger}$, Shi-Juan Mai ${ }^{1,2 \dagger}$, Mei-Yin Zhang ${ }^{1,2}$, Long Huang ${ }^{5}$, Ji Liu ${ }^{1,2}$, Xian-Zi Yang ${ }^{1,2}$, Guo-Liang $\mathrm{Xu}^{1,2,3^{*}}$, Jian-Hua Fu ${ }^{1,2,4^{*}}$ and Hui-Yun Wang ${ }^{1,2^{*}}$ (D)

\begin{abstract}
Background: Long non-coding RNAs (IncRNAs) have been reported to be prognostic biomarkers in many types of cancer. We aimed to identify a IncRNA signature that can predict the prognosis in patients with esophageal squamous cell carcinoma (ESCC).

Methods: Using a custom microarray, we retrospectively analyzed IncRNA expression profiles in 141 samples of ESCC and 81 paired non-cancer specimens from Sun Yat-Sen University Cancer Center (Guangzhou, China), which were used as a training cohort to identify a signature associated with clinical outcomes. Then we conducted quantitative RT-PCR in another 103 samples of ESCC from the same cancer center as an independent cohort to verify the signature.

Results: Microarray analysis showed that there were 338 IncRNAs significantly differentially expressed between ESCC and non-cancer esophagus tissues in the training cohort. From these differentially expressed IncRNAs, we found 16 IncRNAs associated with overall survival (OS) of ESCC patients using Cox regression analysis. Then a 7-IncRNA signature for predicting survival was identified from the 16 IncRNAs, which classified ESCC patients into high-risk and lowrisk groups. Patients with high-risk have shorter OS (HR: 3.555, 95\% Cl 2.195-5.757, p<0.001) and disease-free survival (DFS) (HR: $2.537,95 \% \mathrm{Cl} 1.646-3.909, \mathrm{p}<0.001$ ) when compared with patients with low-risk in the training cohort. In the independent cohort, the 7 IncRNAs were detected by qRT-PCR and used to compute risk score for the patients. The result indicates that patients with high risk also have significantly worse OS ( $H R=2.662,95 \% \mathrm{Cl} 1.588-4.464$, $p<0.001)$ and DFS (HR 2.389, 95\% Cl 1.447-3.946, $p<0.001)$. The univariate and multivariate Cox regression analyses indicate that the signature is an independent factor for predicting survival of patients with ESCC. Combination of the
\end{abstract}

\footnotetext{
*Correspondence: xug|@sysucc.org.cn; fujh@sysucc.org.cn; wanghyun@mail.

sysu.edu.cn

${ }^{\dagger}$ Nuo-Qing Weng, Jun Chi, Jing Wen and Shi-Juan Mai contributed equally

to this work

${ }^{1}$ State Key Laboratory of Oncology in South China, Collaborative

Innovation Center for Cancer Medicine, Sun Yat-Sen University

Cancer Center, 651 Dongfeng East Road, Building 2, Room 704,

Guzngzhou 510060, China

${ }^{3}$ Department of Endoscopy and Laser, Sun Yat-Sen University Cancer

Center, Guangzhou 510060, China

${ }^{4}$ Department of Thoracic Oncology, Sun Yat-Sen University Cancer Center, Guangzhou 510060, China

Full list of author information is available at the end of the article
}

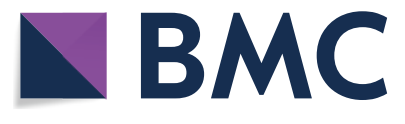

(c) The Author(s) 2020. This article is licensed under a Creative Commons Attribution 4.0 International License, which permits use, sharing, adaptation, distribution and reproduction in any medium or format, as long as you give appropriate credit to the original author(s) and the source, provide a link to the Creative Commons licence, and indicate if changes were made. The images or other third party material in this article are included in the article's Creative Commons licence, unless indicated otherwise in a credit line to the material. If material is not included in the article's Creative Commons licence and your intended use is not permitted by statutory regulation or exceeds the permitted use, you will need to obtain permission directly from the copyright holder. To view a copy of this licence, visit http://creativeco mmons.org/licenses/by/4.0/. The Creative Commons Public Domain Dedication waiver (http://creativecommons.org/publicdomain/ zero/1.0/) applies to the data made available in this article, unless otherwise stated in a credit line to the data. 
signature and TNM staging was more powerful in predicting OS than TNM staging alone in both the training (AUC: 0.772 vs $0.681, p=0.002$ ) and independent cohorts (AUC: 0.772 vs $0.660, p=0.003$ ).

Conclusions: The 7-IncRNA signature is a potential prognostic biomarker in patients with ESCC and may help in treatment decision when combined with the TNM staging system.

Keywords: Esophageal cancer, IncRNA, Expression profile, Survival, TNM stage

\section{Background}

Esophageal cancer (EC) is the eighth most common cancer worldwide, with poor overall 5-year survival rate ranging from 15 to $25 \%$ [1]. EC has two main subtypes: esophageal squamous cell carcinoma (ESCC) predominantly found in Asia, Africa, and South America, and esophageal adenocarcinoma (EAC) predominant in North America and Europe [2]. ESCC accounts approximately $80 \%$ of the EC worldwide, and is a highly aggressive squamous cell carcinoma [3]. Regarding the etiology, the incidence of ESCC is closely related with alcohol and tobacco [4]. The primary method for the diagnosis of EC patients is endoscopy with biopsy [5]. Currently, prognosis and treatment of EC are mainly based on the TNM staging system. However, ESCC patients with same M0 stage according to the 7th/8th edition AJCC staging system have distinct prognoses [6]. Therefore, clinical TNM staging system needs further improvement [7]. Molecular biomarkers have proven to be of great prognostic value for cancer patients $[8,9]$. However, no practical molecular biomarker has been used in the prognostication of EC patients yet, although various biomarkers have been reported in ESCC patients with the rapid development in the molecular biology [10-13]. Clinically, the main treatment options for EC patients include surgery with or without neoadjuvant therapy, and chemoradiotherapy with or without salvage surgery [14]. At present, there is no targeted therapy for EC patients, which has already been applied in patients with other types of cancer $[15,16]$. Although many advances in treatment have been made, the survival of ESCC patients is still disappointedly low. Therefore, there is an urgent need to better understand the biology of ESCC and to identify other more potential molecular biomarkers or targets for prognosis and targeted therapy in the patients.

In recent decades, there have been numerous reports on coding-protein genes as biomarker for prediction of prognosis of ES patients [17-19], but none of them have been used in clinical practice. In the past few years, long non-coding RNAs (lncRNAs), defined as transcripts longer than $200 \mathrm{nt}$ lacking protein-coding capacity, have been reported to have complex biological functions [20-22] and involved in tumorigenesis and metastasis [23]. Some research groups have reported that many single lncRNAs can be used as biomarkers for prediction of survival in ESCC patients [11], but there is only one study on IncRNA expression profile with microarray to date, which identified a prognostic signature (3-lncRNA) in ESCC patients [24]. Therefore, more high-throughput studies are needed to fully discover potential prognostic IncRNA signatures for ESCC patients. In this study, we aimed to explore the lncRNA expression profile in ESCC patients with a custom microarray and identify a lncRNA signature to predict the prognosis of ESCC patients, which may improve the TNM staging system.

\section{Methods \\ Clinical specimens and study design}

In this study, we first collected 141 esophageal squamous cell carcinoma (ESCC) specimens and 81 paired adjacent normal tissues (at least $2 \mathrm{~cm}$ away from the ESCC) from the tissue bank at Sun Yat-Sen University Cancer Center (Guangzhou, China). These samples were obtained from patients who underwent esophagectomy in the Cancer Center between Oct. 2007 and Sep. 2009, which were used as a training cohort. We then obtained another 103 ESCC samples from the patients who were received the esophagectomy between Mar. 2002 and Sep. 2009 at the Department of Thoracic Surgery in the same center, which were used as an independent cohort. The inclusion criteria for this study were as following: (i) pathologically confirmed ESCC; (ii) no pre-operative radiotherapy and/ or chemotherapy; (iii) R0 resection; (iv) no patient died in 1 month after surgery; (v) available frozen tissue samples. The clinical characteristics of all patients in the two cohorts are listed in Table 1. The follow-up time ranged from 3 to 91 months with a median time of 37 months in the training cohort, and 1 month to 150 months with a median time of 43 months in the independent cohort. The overall survival (OS) was defined as the time from the date of surgery to the date of death or last followup, and the disease-free survival (DFS) was defined as the time from the date of surgery to the date of the recurrence in situ, distant metastasis, death or last follow-up. Tumor stage was determined according to the 7 th edition of the TNM classification of the Union for International Cancer Control (UICC). This study was reviewed and approved by the Research Ethics Committee of Sun YatSen University Cancer Center. Written informed consent forms were obtained from every patient prior to surgery. 


\begin{tabular}{|c|c|c|}
\hline Characteristic & Training cohort & Independent cohort \\
\hline \multicolumn{3}{|l|}{ Age } \\
\hline$\leq 59$ & $66(46.8 \%)$ & $54(52.4 \%)$ \\
\hline$>59$ & 75 (53.2\%) & $49(47.6 \%)$ \\
\hline \multicolumn{3}{|l|}{ Gender } \\
\hline Male & 112 (79.4\%) & $74(71.8 \%)$ \\
\hline Female & $29(20.6 \%)$ & $29(28.2 \%)$ \\
\hline \multicolumn{3}{|l|}{ Tobacco use } \\
\hline Yes & 95 (67.4\%) & $66(64.1 \%)$ \\
\hline No & $46(32.6 \%)$ & 37 (35.9\%) \\
\hline \multicolumn{3}{|l|}{ Alcohol use } \\
\hline Yes & $61(43.3 \%)$ & $40(38.8 \%)$ \\
\hline No & $80(56.7 \%)$ & $63(61.2 \%)$ \\
\hline \multicolumn{3}{|l|}{ Tumor location } \\
\hline Upper & $13(9.2 \%)$ & $8(7.8 \%)$ \\
\hline Middle & 95 (67.4\%) & $65(63.1 \%)$ \\
\hline Lower & $33(23.4 \%)$ & $30(29.1 \%)$ \\
\hline \multicolumn{3}{|l|}{ Tumor size } \\
\hline$\leq 4$ & $92(67.3 \%)$ & $63(61.2 \%)$ \\
\hline$>4$ & $47(33.3 \%)$ & $40(38.8 \%)$ \\
\hline \multicolumn{3}{|l|}{ N stage } \\
\hline No & $66(46.8 \%)$ & $64(62.1 \%)$ \\
\hline N1 & $45(31.9 \%)$ & $22(21.4 \%)$ \\
\hline N2 & $20(14.2 \%)$ & $13(12.6 \%)$ \\
\hline N3 & $10(7.1 \%)$ & $4(3.9 \%)$ \\
\hline \multicolumn{3}{|l|}{ TNM stage } \\
\hline । & $4(2.8 \%)$ & $10(9.7 \%)$ \\
\hline$\|$ & $73(51.8 \%)$ & $57(55.3 \%)$ \\
\hline III & $64(45.4 \%)$ & $36(35.0 \%)$ \\
\hline
\end{tabular}

RNA isolation, labeling and microarray hybridization Total RNAs were isolated from fresh-frozen ESCC and noncancerous esophagus tissues using TRIzol reagent (Invitrogen) according to the manufacturer's instructions, and the concentration and purity of total RNAs were estimated with a NanoDrop 2000 spectrophotometer (Thermo Scientific, Wilmington, DE, USA). If the ratio of A260/A280 was between 1.9 and 2.1, the total RNA was accepted for the subsequent experiments.

To fabricate the custom IncRNA microarray, we first selected 2412 lncRNAs from literatures and public databases for designing the probes. Then the lncRNA probes were synthesized by Invitrogen company (Shanghai, China). The probes were mixed well with printing buffer $(1: 1)$ and printed on the cleaned slides in SmartArray ${ }^{\mathrm{TM}}$ 136 printer (CapitalBio Inc., Beijing, China) as previously described $[25,26]$. RNA labeling and microarray hybridization were conducted according to the published protocols $[26,27]$ with minor adjustments. Briefly, $2 \mu \mathrm{g}$ of total RNAs from the training cohort were reverse transcribed using random primers and Cy5-dUTP or Cy3dUTP with GoScript ${ }^{\mathrm{TM}}$ Reverse Transcription system (Promega) in a total reaction volume of $20 \mu$ l. The labeled cDNA products were purified and eluted in $100 \mu \mathrm{L}$, and thermally vacuum-evaporated to $25 \mu \mathrm{l}$. And then the labeled cDNAs were mixed with $25 \mu \mathrm{l}$ hybridization solution containing $5 \times$ Denhart's, $0.5 \%$ SDS and $3 \times$ SSC. After hybridization solution was added onto the microarray, hybridization was performed in a Hybridization Chamber (Corning Inc.) at a temperature of $45^{\circ} \mathrm{C}$ for 16 to $18 \mathrm{~h}$. After washing with consecutive solutions, the microarray was scanned by the LuxScan $10 \mathrm{~K}$ Microarray Scanner (CapitalBio, Beijing, China) at PMT 950. GenePix Pro 6.0 software (Axon Instruments, Foster City, CA, USA) was used to digitize the images.

Microarray raw data were subtracted by the background and normalized with quantile method [28], and then the normalized data were used for further analysis. The microarray data have been uploaded into the Gene Expression Omnibus Public Database at the National Center for Biotechnology Information, USA, and the accession number is GSE92986 (https://www.ncbi.nlm. nih.gov/geo/query/acc.cgi?acc=GSE92986).

\section{Real time quantitative reverse transcription-PCR}

For the reverse transcription, total RNAs $(2 \mu \mathrm{g})$ in a $20 \mu$ l volume were reversely transcribed with GoScript ${ }^{\mathrm{TM}}$ Reverse Transcription system (Promega). Then the quantitative PCR reaction was carried out with $1 \mu \mathrm{l}$ of cDNA product, the specific primers and Platinum SYBR Green qPCR SuperMix-UDG reagents (Invitrogen, Carlsbad, CA, USA) using the following reaction cycle: $95{ }^{\circ} \mathrm{C}$ for $120 \mathrm{~s}, 45$ cycles of $95^{\circ} \mathrm{C}$ for $15 \mathrm{~s}, 60^{\circ} \mathrm{C}$ for $60 \mathrm{~s}$ and a dissociation stage. The PCR primer sequences were listed in Additional file 1: Table S1, and the qPCR reaction for each sample was conducted in triplicate. The relative expression levels of lncRNAs were normalized by GAPDH (reference gene) expression and the median value of a given lncRNA's expression in all samples, and presented as $2^{-\Delta \Delta C T}$.

\section{Bioinformatic analysis}

To investigate the biological functions of the 7 lncRNAs, we downloaded the 7 lncRNAs-related transcription factors (TF) from the published lncRNA-TF interactome database RegRNA2.0. Then, the target genes, which are transcribed by the TFs related to the seven lncRNAs, were downloaded from Harmonizome database. The Gene ontology (GO) and Kyoto Encyclopedia of Genes and Genomes (KEGG) pathway enrichment analysis were 
performed on these target genes to identify the biological processes associated with these seven lncRNAs. The clusterProfiler $\mathrm{R}$ package was used to perform the analysis and the $P$ value less than 0.05 was defined as significant.

\section{Statistical analysis}

Significance analysis of microarrays (SAM) program was conducted to identify differentially expressed lncRNAs between 141 samples of ESCC and 81 paired non-cancer specimens with a threshold of 1.25 fold change between the two groups with $P$ values less than 0.1 in the training cohort. Univariate Cox regression analysis was used to identify the lncRNAs that were significantly associated with OS $(P<0.1)$ from the differentially expressed ones. Then we constructed a formula to calculate a risk score for each patients using all lncRNAs that were correlated with survival according to the method previously reported [29]: Risk score $=\sum_{i=1}^{n}[\operatorname{Expression}(\mathrm{n}) \times \operatorname{Exp}(\mathrm{Bn})]$. In this formula, the expression (n) is the expression value of every lncRNAs detected in each sample by microarray and the $\operatorname{Exp}(\mathrm{Bn})$ is the coefficient of each lncRNA obtained from Univariate Cox regression analysis. The risk score was calculated for each patients using the formula. The median value of the risk scores of all patients was employed to divide the patients into high-and lowrisk groups in the training cohort. Kaplan-Meier method and log-rank test were employed to compare the survival of the two groups. Then we removed one lncRNA at a time from the survival lncRNAs (n) to compose n combinations (n-1 lncRNAs), and re-calculated the risk score for patients with all the combinations (risk score formulas), divided patients into high- and low-risk groups based on the risk score of each patient, and carried out the survival analysis again based on all the different lncRNA combinations. One lncRNA would be deleted if it is not a component of the best lncRNA combination (with the smallest $\mathrm{p}$ value in the survival analysis). Next, we composed n-1 combinations ( $\mathrm{n}-2$ lncRNAs) again and did the same analysis until 2-lncRNA combination. Finally, we compared all results and found the best combination (signature), which would be used for the further analysis.

$X^{2}$ test or Fisher's exact test was applied to assess the relationship between lncRNA relative expression and clinical characteristics. The Kaplan-Meier method and the log-rank test were applied to assess the OS and DFS. The lncRNA signature, age, gender, pathologic grade, TNM stage, radiotherapy, chemotherapy, alcohol use, and tobacco use were analyzed as covariates with multivariate Cox regression by a forward LR approach in order to determine which feature(s) was an independent factor for OS and DFS. We then set up a new model that combined all the independent prognostic factors to predict the survival of each patient. Receiver operating characteristic (ROC) curves were employed to compare the sensitivity and specificity of TNM stage, lncRNA signature and the combination of both. Significance was defined as $p<0.05$. The statistical analyses were done with SPSS version 23.0, GraphPad Prism 6.0c and MedCalc Version 11.4.2.

\section{Results \\ LncRNA expression profiles of ESCC tissues}

To profile lncRNA expression in ESCC tissues, we used the custom lncRNA microarray (Additional file 2: Fig. S1A) to detect 141 ESCCs and 81 paired esophagus tissues in the training cohort. After subtracting background and normalizing the hybridization intensity, we analyzed the lncRNA expression profile with SAM program. The result reveals that 338 lncRNAs are differentially expressed between ESCC and adjacent normal tissues (fold change $>1.25, \mathrm{p}<0.1$ ). Of these 338 lncRNAs, 211 are upregulated and the other 127 are downregulated in ESCC tissues.

To validate lncRNA expression levels detected by the microarray analysis, we randomly selected two upregulated lncRNAs (ASLNC11164 and BQ376030) and two downregulated IncRNAs (XLOC_001061 and RP11473M20.9) in ESCC tissues and detected them by quantitative RT-PCR in 40 pairs of ESCC and noncancerous esophagus tissues randomly chosen from the training cohort. As shown in Additional file 2: Fig. S1B, the expression levels of the 4 lncRNAs detected by qRT-PCR are consistent with those detected by microarray, suggesting that microarray data are repeatable and reliable and can be used for the further analysis.

\section{Identification of a 7-IncRNA signature for predicting the survival of ESCC patients in the training cohort}

To explore the clinical role of IncRNA in ESCC patient prognosis, we first screened the 338 differentially expressed lncRNAs to find ones associated with survival using Univariate Cox regression analysis. With this analysis, we found 16 lncRNAs that were significantly associated with OS in the training cohort (Additional file 1: Table S2). Then we used the approach aforementioned in Method section to determine a lncRNA combination from the 16 lncRNAs, which can predict the survival of ESCC patients with highest efficiency. Finally, the best 7-lncRNA signature for predicting survival of patients was identified. The formula consists of a linear combination of the expression level of 7 lncRNAs weighted by Cox regression coefficient:

Risk score $=(0.155 \times$ expression value of BQ376030 $+0.112 \times$ expression value of 
ASLNC11164 $+0.125 \times$ expression BF894811+0.155 $\times$ expression value of RP11473M20.9-0.182 $\times$ expression value of XLOC_007869-0.15 $\times$ XLOC_006476 $-0.172 \times$ expression value of CK327190).

Among these 7 lncRNAs, four are risk factors for survival and the other three are protective ones (Additional file 1: Table S2). The formula was employed to compute a risk score for each patient in the training cohort. With the risk score, patients were divided into high- and lowrisk groups according to the median risk score in this cohort. Then we explored the relationship of the signature risk with clinical characteristics in ESCC patients, and found that patients with high risk had significantly higher lymph node metastasis rate and mortality rate (Table 2). Kaplan-Meier analysis revealed that patients in the high-risk group had a significantly shorter OS and DFS than those in the low-risk group (both $p<0.001$, Fig. 1a, b).

\section{Confirmation of the 7-IncRNA prognostic signature in the independent cohort with qRT-PCR}

In order to validate the reliability and repeatability of the 7-lncRNA prognostic signature in ESCC patients, we collected another 103 ESCC samples as a independent cohort in the same cancer center and extracted total

Table 2 The relationships of 7-IncRNA signature and Clinical characteristics of ESCC patients in the training cohort and independent cohort

\begin{tabular}{|c|c|c|c|c|c|c|}
\hline \multirow[t]{3}{*}{ Characteristics } & \multicolumn{2}{|c|}{ Training cohort $(\mathrm{N}=141)$} & \multirow[t]{3}{*}{$p$ value } & \multicolumn{2}{|c|}{ Independent cohort $(\mathrm{N}=103)$} & \multirow[t]{3}{*}{ p value } \\
\hline & Low risk & High risk & & Low risk & High risk & \\
\hline & n (\%) & n (\%) & & n (\%) & n (\%) & \\
\hline \multicolumn{7}{|l|}{ Age } \\
\hline$\leq 59$ & $37(52.1 \%)$ & $35(50.0 \%)$ & 0.802 & $29(55.8 \%)$ & $25(49.0 \%)$ & 0.493 \\
\hline$>59$ & $34(47.9 \%)$ & $35(50.0 \%)$ & & $23(44.2 \%)$ & $26(51.0 \%)$ & \\
\hline \multicolumn{7}{|l|}{ Gender } \\
\hline Male & $58(81.7 \%)$ & $54(77.1 \%)$ & 0.500 & $36(69.2 \%)$ & $38(74.5 \%)$ & 0.660 \\
\hline Female & $13(18.3 \%)$ & $16(22.9 \%)$ & & $16(30.8 \%)$ & $13(25.5 \%)$ & \\
\hline \multicolumn{7}{|l|}{ Tobacco use } \\
\hline Yes & $49(69.0 \%)$ & $46(65.7 \%)$ & 0.680 & $31(59.6 \%)$ & $35(64.1 \%)$ & 0.413 \\
\hline No & $22(31.0 \%)$ & $24(34.3 \%)$ & & $21(40.4 \%)$ & $16(35.9 \%)$ & \\
\hline \multicolumn{7}{|l|}{ Alcohol use } \\
\hline Yes & $34(47.9 \%)$ & $27(38.6 \%)$ & 0.260 & $22(42.3 \%)$ & $18(35.3 \%)$ & 0.546 \\
\hline No & $37(52.1 \%)$ & $43(61.4 \%)$ & & $30(57.7 \%)$ & $33(64.7 \%)$ & \\
\hline \multicolumn{7}{|l|}{ Tumor location } \\
\hline Upper & $6(8.5 \%)$ & $7(10.0 \%)$ & 0.510 & $2(3.8 \%)$ & $6(11.8 \%)$ & 0.210 \\
\hline Middle & $51(71.8 \%)$ & 44 (62.9\%) & & $32(61.5 \%)$ & $33(64.7 \%)$ & \\
\hline Lower & 14 (19.7\%) & $19(27.1 \%)$ & & $18(34.6 \%)$ & $12(23.5 \%)$ & \\
\hline \multicolumn{7}{|l|}{ Tumor size } \\
\hline$\leq 4$ & 48 (67.6\%) & 46 (65.7\%) & 0.810 & $33(63.5 \%)$ & $30(58.8 \%)$ & 0.629 \\
\hline$>4$ & 23 (32.4\%) & $24(32.3 \%)$ & & 19 (36.5\%) & $21(41.2 \%)$ & \\
\hline \multicolumn{7}{|l|}{ N stage } \\
\hline NO & $40(56.3 \%)$ & $26(37.1 \%)$ & 0.050 & $35(67.3 \%)$ & $29(56.9 \%)$ & 0.240 \\
\hline N1 & $22(31.0 \%)$ & 23 (32.9\%) & & $11(21.2 \%)$ & $11(21.6 \%)$ & \\
\hline $\mathrm{N} 2$ & $6(8.5 \%)$ & 14 (20.0\%) & & $6(11.5 \%)$ & $7(13.7 \%)$ & \\
\hline N3 & $3(4.2 \%)$ & $7(10.0 \%)$ & & $0(0.0 \%)$ & $4(7.8 \%)$ & \\
\hline \multicolumn{7}{|l|}{ TNM stage } \\
\hline । & $2(2.81 \%)$ & $2(2.86 \%)$ & 0.579 & $8(15.4 \%)$ & $2(3.9 \%)$ & 0.131 \\
\hline$\|$ & $34(47.9 \%)$ & $39(55.7 \%)$ & & $28(53.8 \%)$ & $29(56.9 \%)$ & \\
\hline III & 35 (49.3\%) & $28(40.0 \%)$ & & $16(30.8 \%)$ & $20(39.2 \%)$ & \\
\hline \multicolumn{7}{|l|}{ Survival status } \\
\hline Survival cases & $50(70.4 \%)$ & $20(28.6 \%)$ & $<0.001$ & 32 (61.5\%) & $11(21.6 \%)$ & $<0.001$ \\
\hline Death cases & $21(29.6 \%)$ & $50(71.4 \%)$ & & 20 (38.5\%) & 40 (78.4\%) & \\
\hline
\end{tabular}



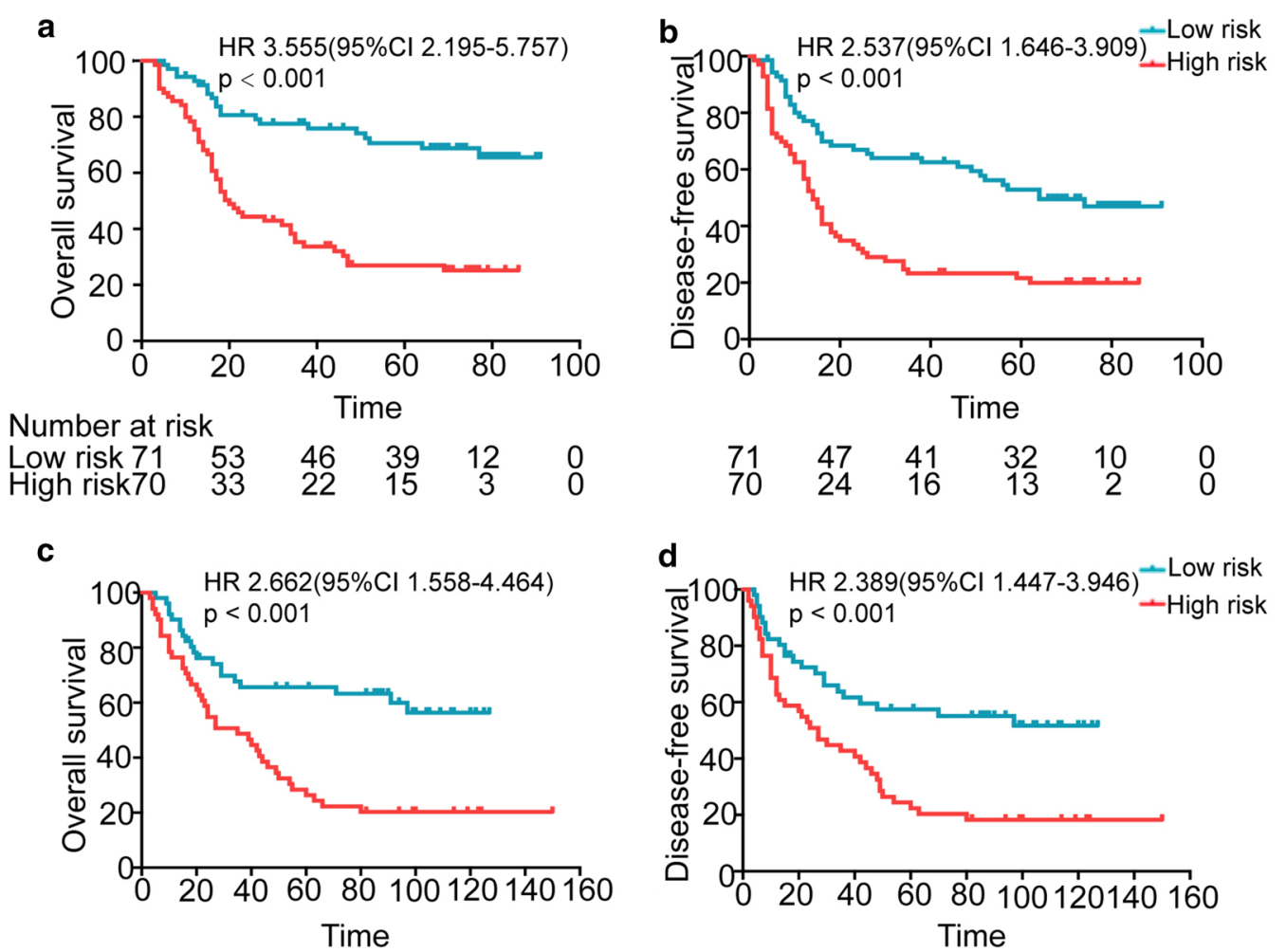

Number at risk

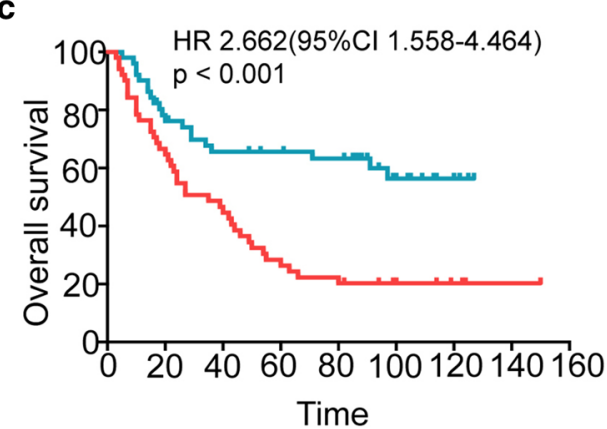

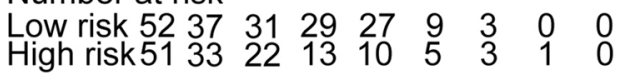

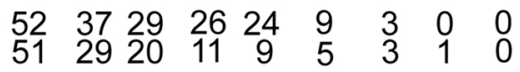

Fig. 1 The 7-InCRNA signature is associated with survivals of ESCC patients in the training and independent cohorts. The risk score was calculated for each patient according to the 7-IncRNA signature and the patients were divided into a high- or low-risk group based on their risk score. Then Kaplan-Meier survival analysis was performed on the patients. a Overall survival (OS) curves of 141 patients with high-risk or low-risk in the training cohort. b Disease-free survival (DFS) curves of 141 patients in the training cohort. c OS curves of 103 patients with high-risk or low-risk in the independent cohort. d DFS curves of 103 patients with high-risk or low-risk in the independent cohort. Note: in the Kaplan-Meier survival curves, the survival time unit is months

RNA from these samples. The expression levels of the 7 lncRNAs were measured by qRT-PCR in these RNA samples. The qRT-PCR data were presented as $2^{-\Delta \Delta C T}$ and analyzed with Cox regression. Then we constructed a new risk score formula with the same method used in the training cohort:

Risk score $=(0.028 \times$ expression value of BQ376030 $+0.053 \times$ expression value of ASLNC11164 $+0.049 \times$ expression value of BF894811 $+0.031 \times$ expression value of RP11473M20.9-0.058 $\times$ expression value of XLOC_00 $7869-0.116 \times$ XLOC_006476 $-0.061 \times$ expression value of CK327190).

With this formula, we computed a risk score for every patient and divided the patients into low and high risk groups according to the median risk score in the independent cohort. Survival analysis revealed that high-risk patients showed notably poorer prognosis than low-risk ones in the independent cohort (both $P<0.001$ in OS and DFS, Fig. 1c, d), suggesting that the 7-lncRNA signature is a reliable and repeatable survival predictor in patients with ESCC.

\section{The 7-IncRNA signature is an independent prognostic factor in patients with ESCC}

We then wanted know whether the 7-lncRNA signature was an independent predictor for patients with ESCC. To this end, we conducted univariate and multivariate Cox proportional hazards regression analysis on the 7-lncRNA signature and clinical features in the training and independent cohorts, respectively. The results show that 7-lncRNA signature and TNM stage are independent prognostic factors for OS and DFS of patients in both the training (Table 3 ) and independent (Table 4) cohorts. The hazard ratio (HR) for tumor-related death in patients with high risk was about 2.5 times higher than those with low risk. Furthermore, we analyzed the prognostic role of the 7-lncRNA signature in all ESCC patients of the two cohorts. The result also exhibits that the 7-lncRNA 
Table 3 Univariate and multivariable Cox analysis of the effects of 7-IncRNA signature and clinical characteristics on overall survival in the training cohort and independent cohort

\begin{tabular}{|c|c|c|c|c|}
\hline \multirow[t]{2}{*}{ Variables } & \multicolumn{2}{|c|}{ Univariable analysis } & \multicolumn{2}{|c|}{ Multivariable analysis } \\
\hline & $\mathrm{HR}(95 \% \mathrm{Cl})$ & p value & $\mathrm{HR}(95 \% \mathrm{Cl})$ & $\mathrm{p}$ value \\
\hline \multicolumn{5}{|l|}{ Training cohort } \\
\hline Age $(\leq 59$ vs $>59)$ & $1.48(0.93-2.37)$ & 0.101 & & \\
\hline Gender (Male vs female) & $1.04(0.60-1.82)$ & 0.878 & & \\
\hline Tobacco use (Y vs N) & $1.08(0.66-1.78)$ & 0.752 & & \\
\hline Alcohol use (Y vs N) & $1.01(0.63-1.62)$ & 0.981 & & \\
\hline Tumor location (U vs M vs L) & $1.41(0.94-2.41)$ & 0.095 & & \\
\hline Differentiation (H vs Mt vs L) & $1.34(0.94-1.89)$ & 0.104 & & \\
\hline TNM stage (III vs I, II) & $3.17(1.94-5.20)$ & $<0.001$ & $2.80(1.80-4.61)$ & $<0.001$ \\
\hline 7-LncRNA signature (H-risk vs L- risk) & $3.54(2.11-5.92)$ & $<0.001$ & $3.16(1.90-5.31)$ & $<0.001$ \\
\hline \multicolumn{5}{|l|}{ Independent cohort } \\
\hline Age $(\leq 59$ vs $>59)$ & $1.02(0.62-1.70)$ & 0.926 & & \\
\hline Gender (Male vs female) & $1.22(0.70-2.12)$ & 0.486 & & \\
\hline Tobacco use (Y vs N) & $1.12(0.65-1.92)$ & 0.682 & & \\
\hline Alcohol use (Y vs N) & $0.96(0.57-1.61)$ & 0.864 & & \\
\hline Tumor location (U vs M vs L) & $0.94(0.60-1.47)$ & 0.784 & & \\
\hline Differentiation (H, Mt vs L) & $1.40(0.97-2.01)$ & 0.069 & & \\
\hline TNM stage (III vs I, II) & $3.25(1.94-5.46)$ & $<0.001$ & $3.08(1.83-5.18)$ & $<0.001$ \\
\hline 7-LncRNA signature (H-risk vs L-risk) & $2.67(1.55-4.58)$ & $<0.001$ & $2.51(1.46-4.33)$ & 0.001 \\
\hline
\end{tabular}

$Y$ yes, $N$ no, $U$ upper, $M$ middle, $L$ low, $H$ high, $M t$ moderate, $H$-risk high-risk, L-risk low-risk

Table 4 Univariate and multivariable Cox analysis of the effects of 7-IncRNA signature and clinical characteristics on disease-free survival in the independent cohort

\begin{tabular}{|c|c|c|c|c|}
\hline \multirow[t]{2}{*}{ Variables } & \multicolumn{2}{|c|}{ Univariable analysis } & \multicolumn{2}{|c|}{ Multivariable analysis } \\
\hline & $\mathrm{HR}(95 \% \mathrm{Cl})$ & p value & $\mathrm{HR}(95 \% \mathrm{Cl})$ & $p$ value \\
\hline \multicolumn{5}{|l|}{ Training cohort } \\
\hline Age ( $\leq 59$ vs $>59)$ & $0.96(0.65-1.48)$ & 0.906 & & \\
\hline Gender (Male vs female) & $0.78(0.46-1.32)$ & 0.357 & & \\
\hline Tobacco use (Y vs N) & $1.30(0.82-2.05)$ & 0.265 & & \\
\hline Alcohol use (Y vs N) & $1.10(0.73-1.67)$ & 0.657 & & \\
\hline Tumor location (U vs M vs L) & $1.26(0.86-1.85)$ & 0.238 & & \\
\hline Differentiation (H vs Mt vs L) & $2.49(1.63-3.81)$ & $<0.001$ & $2.23(1.45-3.44)$ & $<0.001$ \\
\hline TNM stage (III vs I, II) & $2.41(1.57-3.70)$ & $<0.001$ & $1.93(1.24-3.01)$ & 0.003 \\
\hline LncRNA signature (H-risk vs L- risk) & $2.36(1.41-3.95)$ & $<0.001$ & $2.28(1.36-3.82)$ & 0.002 \\
\hline \multicolumn{5}{|l|}{ Independent cohort } \\
\hline Age ( $\leq 59$ vs $>59)$ & $1.04(0.64-1.70)$ & 0.868 & & \\
\hline Gender (Male vs female) & $1.14(0.67-1.95)$ & 0.632 & & \\
\hline Tobacco use (Y vs N) & $1.03(0.62-1.73)$ & 0.901 & & \\
\hline Alcohol use (Y vs N) & $1.05(0.63-1.73)$ & 0.858 & & \\
\hline Tumor location (U vs M vs L) & $0.98(0.64-1.51)$ & 0.941 & & \\
\hline Differentiation (H vs Mt vs Low) & $1.33(0.94-1.89)$ & 0.104 & & \\
\hline TNM stage (III vs I, II) & $2.66(1.60-4.40)$ & $<0.001$ & $2.58(1.55-4.27)$ & $<0.001$ \\
\hline LncRNA signature (H-risk vs L-risk) & $2.36(1.41-3.95)$ & 0.001 & $2.28(1.36-3.82)$ & 0.002 \\
\hline
\end{tabular}

$Y$ yes, $N$ no, $U$ upper, $M$ middle, $L$ low, $H$ high, $M t$ moderate, $H$-risk high-risk, L-risk low-risk 
signature is an independent prognostic factor for OS and DFS in all ESCC patients of the two cohorts (Additional file 1: Table S3). To further confirm that the 7-lncRNA signature is an prognostic predictor independent from TNM staging system, we analyzed the effect of 7-lncRNA signature on survival in different TNM stages in the training cohort. Since the number of patients with stage I is too small, we deleted these patients from the subsequent analysis. The results demonstrate that 7-lncRNA signature is a significant predictor for OS and DFS in patients with TNM stage II or III in the training cohort (Fig. 2), respectively, demonstrating that this signature is a prognostic factor independent of clinical stage and can offer additional information to evaluate survival of ESCC patients.
The 7-IncRNA signature is able to improve the prognostic power of the TNM staging system in ESCC patients

TNM staging system is a standard method for predicting survival in patients with malignant tumors and determining the treatment strategy. However, TNM staging system is not satisfactory enough to predict outcomes in ESCC patients. As mentioned above, ESCC patients with the same M0 stage may have significantly different prognoses [6]. Therefore, we tried to improve the prognostic power of TNM staging system in ESCC patients by incorporating the 7-lncRNA signature. For the same reason aforementioned, we removed the patients with stage I from this analysis. The combined risk score model was established with the following method: the signature score (low risk score $=0$, and high risk score $=1$ ) plus the TNM stage score (stage $I I=1$, stage $I I I=2$ ). With this combined risk score model, patients were classified to a low- (1 score), moderate- (2 score) and high-risk (3
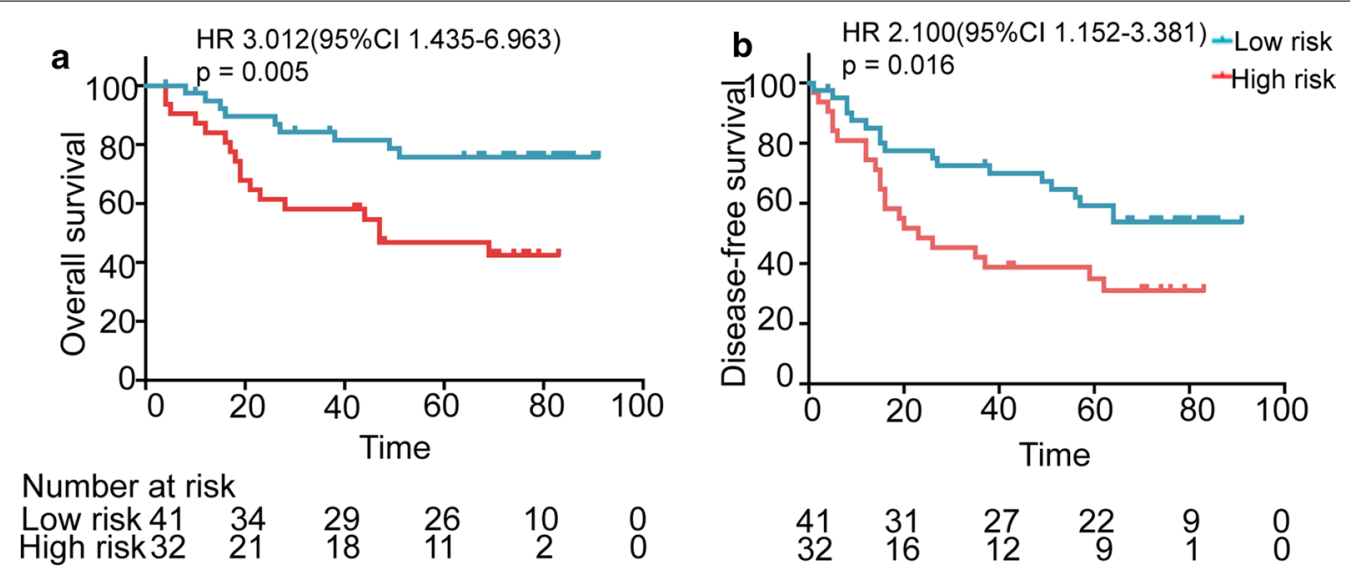

C

d
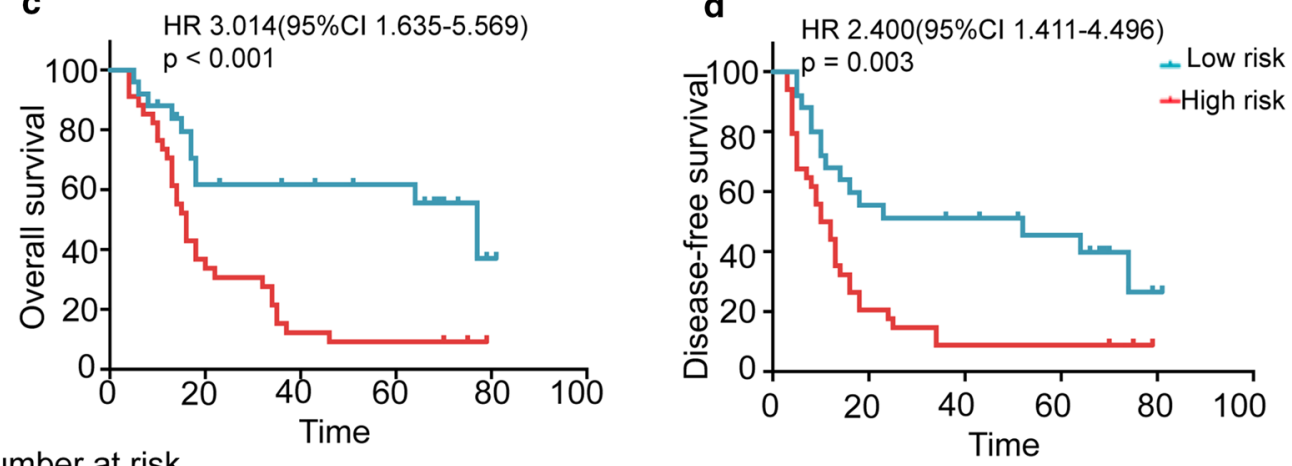

Number at risk

$\begin{array}{lccccc}\text { Low risk } 25 & 14 & 12 & 10 & 1 & 0 \\ \text { High risk34 } & 11 & 4 & 11 & 0 & 0\end{array}$

$\begin{array}{llllll}25 & 13 & 11 & 8 & 1 & 0 \\ 34 & 7 & 3 & 3 & 0 & 0\end{array}$

Fig. 2 The 7-IncRNA signature can predict distinct survivals of ESCC patients with same TNM stage in the training cohort. The patients with same stage (II or III) were defined as high- or low-risk by the 7-IncRNA signature risk score and then analyzed with Kaplan-Meier survival curves. a Overall survival (OS) curves of 73 patients with high-risk or low-risk in the cases with TNM stage II in the training cohort. b Disease-free survival (DFS) curves in 73 patients with high-risk or low-risk in the cases with TNM stage II in the training cohort. c OS curves in 59 patients with high-risk or low-risk in the cases with TNM stage III in the training cohort. d DFS curves in 59 patients with high-risk or low-risk in the cases with TNM stage II in the training cohort. Note: in the Kaplan-Meier survival curves, the survival time unit is months 
score) groups based on the combined risk score of every patients. The Kaplan-Meier survival analysis reveals that the patients with low-, medium- or high-risk have distinctly different OS (Fig. 3) and DFS (Additional file 2: Fig. S2) in the training cohort, independent cohort and combined cohort, indicating that this combined risk model can provide more accurate survival prediction with three different risk levels.
To compare the performance of the combined risk score model with that of TNM staging system and 7-lncRNA signature, we performed receiver operating characteristic (ROC) analysis. The result reveals that the combined score model has better predictive performance in predicting OS (AUC: 0.772) and DFS (AUC: 0.727) than the 7-lncRNA signature (OS AUC: 0.709; DFS AUC: 0.658 ) and TNM stage alone (OS AUC: 0.681; DFS AUC: 0.667) in the training cohort (Fig. 4a, b). In a
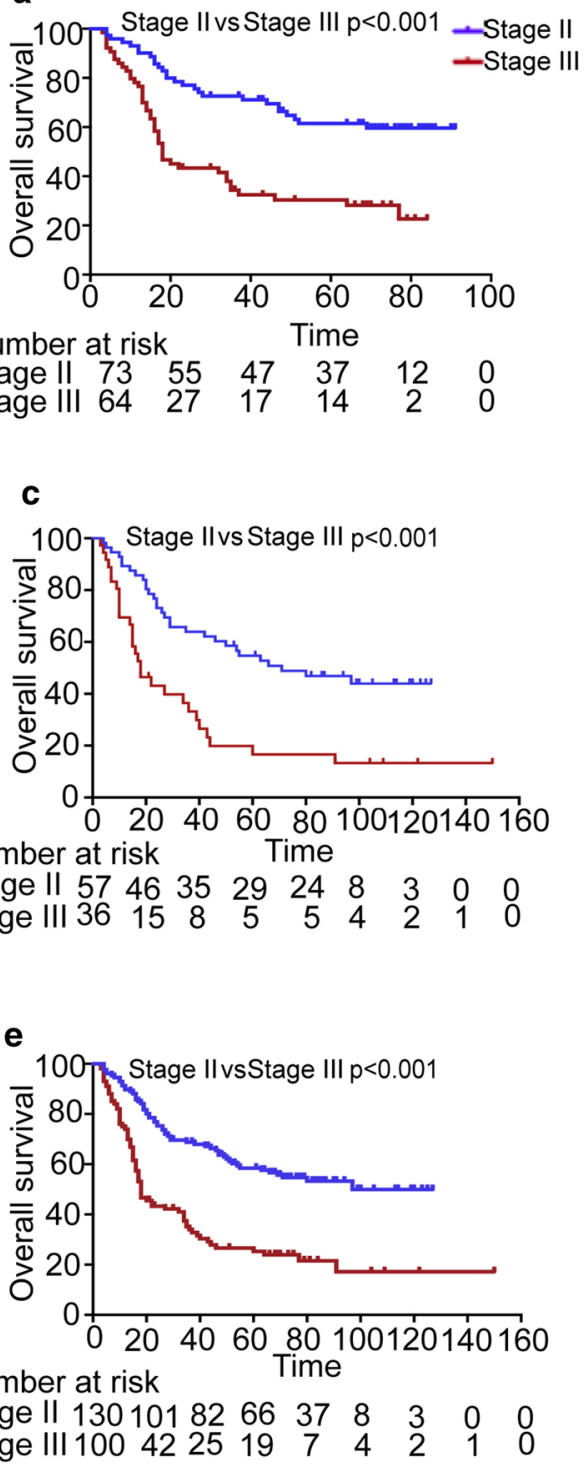

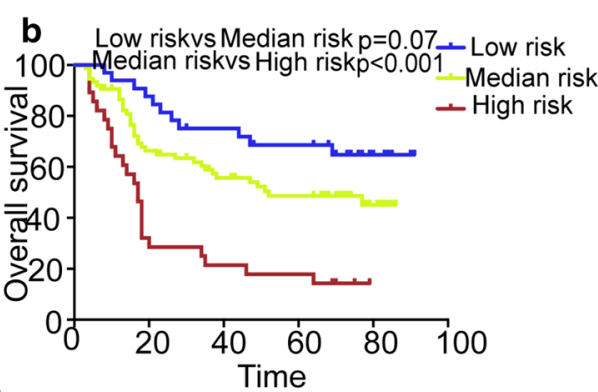

$\begin{array}{cccccc}\text { Low } 36 & 30 & 25 & 21 & 8 & 0 \\ \text { Median } 75 & 46 & 35 & 26 & 7 & 0 \\ \text { High 26 } & 8 & 6 & 5 & 0 & 0\end{array}$

d

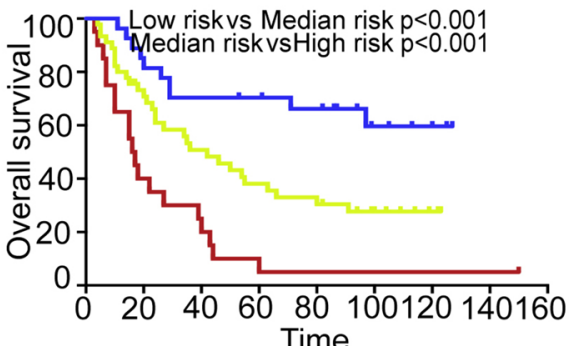

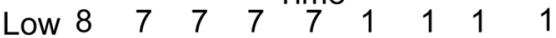
$\begin{array}{rllllllll}\text { Median } 30 & 24 & 21 & 18 & 17 & 6 & 3 & 0 & 0 \\ \text { High 45 } & 30 & 20 & 15 & 12 & 6 & 2 & 0 & 0\end{array}$

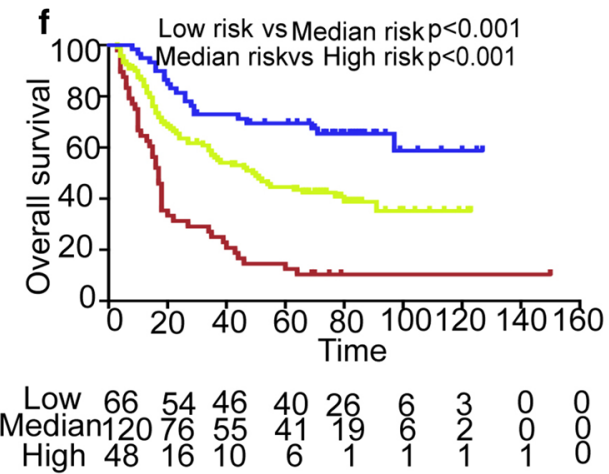

Fig. 3 The 7-IncRNA signature improves survival prediction of TNM staging system in ESCC patients. The ESCC patients were defined as low-, moderate- and high-risk by the combination model of 7-IncRNA signature and TNM staging system, and then the survivals of these patients were analyzed with Kaplan-Meier curves and compared with the survivals predicted by TNM staging system. a Overall survival (OS) of patients with TNM stage II or III in the training cohort; $\mathbf{b}$ OS of patients with low-, moderate- or high-risk score in the training cohort. $\mathbf{c}$ OS of patients with TNM stage II or III in the independent cohort; $\mathbf{d}$ OS of patients with low-, moderate- or high-risk score in the independent cohort; $\mathbf{e}$ OS of patients with TNM stage II or III in the combination of two cohorts. f OS of patients with low-, moderate- or high-risk score in combination of two cohorts. In the Kaplan-Meier survival curves, the survival time unit is months 


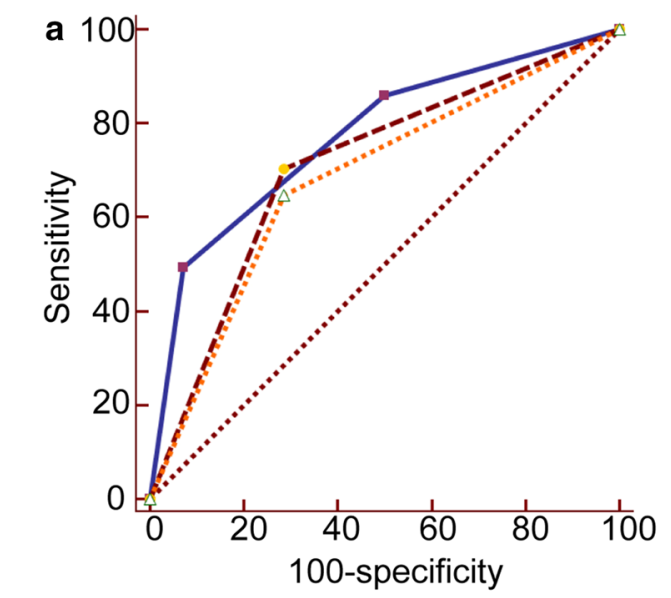

AUC $\quad 95 \% \mathrm{Cl}$ p value

TNM+IncRNA

LncRNA

TNM stage

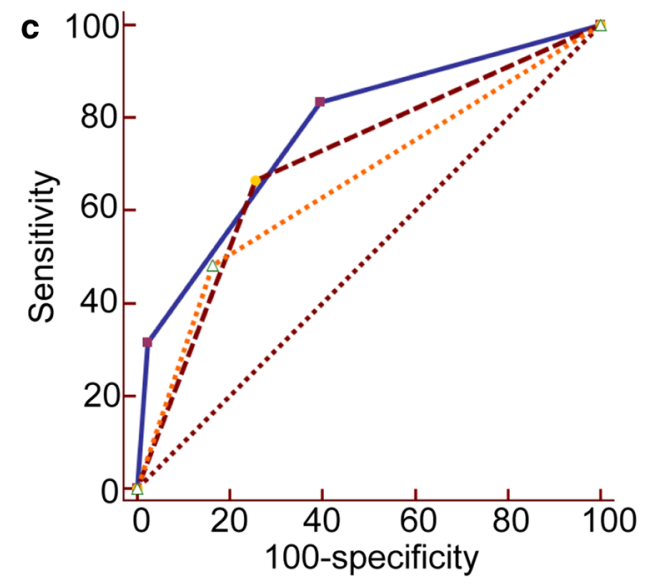

$\begin{array}{llcl} & \text { AUC } & 95 \% \mathrm{Cl} \quad \mathrm{p} \text { value } \\ \text { TNM+IncRNA } & 0.772 & 0.705-0.869 \\ \text { LncRNA } & 0.705 & 0.607-0.791 & 0.045 \\ \text { TNM stage } & 0.660 & 0.615-0.798 & 0.003\end{array}$

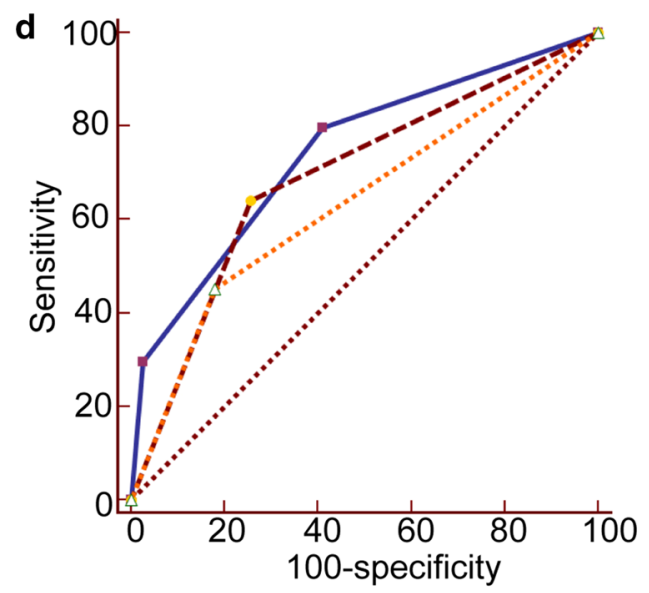

AUC $95 \% \mathrm{Cl}$ p value

$0.744 \quad 0.649-0.825$

$0.692 \quad 0.593-0.7790 .128$

$\begin{array}{lll}0.637 & 0.536-0.729 & 0.005\end{array}$
AUC $95 \% \mathrm{Cl}$ p value
$0.727 \quad 0.647-0.798$
$\begin{array}{llll}0.658 & 0.574-0.736 & 0.024\end{array}$
$\begin{array}{lll}0.667 & 0.583-0.744 & 0.051\end{array}$

Fig. 4 Comparisons of the performances of survival predictions made by the 7-IncRNA signature, TNM stage and combined model of the signature and TNM stage. The performances of survival predictions made by the three methods were compared using receiver operating characteristic (ROC) analysis. a ROC curves of the 7-InCRNA signature, TNM stage and combined model for overall survival (OS) prediction in the training cohort. $\mathbf{b}$ ROC curves of the three methods for disease-free survival (DFS) prediction in the training cohort. $\mathbf{c}$ ROC curves of the three methods for OS prediction in the independent cohort. $\mathbf{d}$ ROC curves of the three methods for DFS prediction in the independent cohort

the independent cohorts, we obtained similar results (Fig. 4c, d). We got much better predictive performance of the combined score model when the two cohorts were combined (Additional file 2: Fig. S3). These above results demonstrate that the 7-IncRNA signature can provide additional prognostic information to clinician and improve the predictive performance of TNM staging system in the evaluation of survival of ESCC patients.
The 7 IncRNAs of the signature is likely to correlate with tumour-associated biological functions in ESCC

Finally, we hope to explore the potential role of the seven lncRNAs of this signature in ESCC development and progression. In our study, we could not conduct the lncRNA-gene interaction network because of lack of coexpressed lncRNA-mRNA data in ESCC. As we know, however, lncRNA can bind to transcription factors (TF) and regulate their transcriptional activity. To this end, we 
identified that 44 TFs could be bound by the 7 lncRNAs in the public lncRNA-TF interactome database RegRNA2.0, and 17,362 target genes can be regulated by the $44 \mathrm{TFs}$ in Harmonizome database. Then, we found that 555 of 17,362 genes were overlapped with 575 enriched genes in esophageal cancer obtained from TCGA database as shown in Venn diagram (Additional file 2: Fig S4). Next, we conducted GO enrichment analysis and the KEGG pathway. In the GO enrichment analysis, these genes are mainly enriched in the cell-cell signaling by Wnt, Wnt signaling pathway and autophagy (Fig. 5a). In the KEGG pathway analysis, the 7 lncRNAs are primarily involved in MAPK signaling pathway, proteoglycans in cancer, and AMPK signaling pathway (Fig. $5 \mathrm{~b}$ ). These results suggest that the lncRNAs signature is not only a prognostic factor but also likely regulates the cancer-related pathways in the progression of esophageal cancer.

\section{Discussion}

As the standard method for predicting survival in human cancer, TNM staging system plays a critical role in treatment choice for ESCC patients. TNM staging system is based on the size of primary cancer and anatomical site of the primary and metastatic cancers. However, malignant behavior is mainly determined by the molecular and genetic characteristics of the cancer. Consequently, survival prediction solely based on the tumor size and anatomical site is not fully satisfactory yet to physicians in clinical practice [30,31], suggesting that evaluation of prognosis should be based on both the TNM stage system and molecular/genetic characteristics of the cancer. Gene expression profile is one of the most comprehensive ways in understanding the molecular changes in cancer. In recent decades, gene expression profiles have been explored in nearly all types of cancer due to advancements in high throughput technologies (microarray, deep-sequencing, PCR and others), and many gene signatures have been identified for predicting survival of patients with cancer [32-34]. For example, a 70-gene signature has been used for predicting patients' survival of in breast cancer [35] and a 42-gene signature has been used for prediction of disease relapse in early stage colon cancer [36]. In esophageal cancer, many studies on gene expression profile have been reported, but the case number in these studies is small (the biggest being 89 cases) [37] and no gene signature has been applied in this disease at present. Recently, non-coding RNA (including microRNA [miRNA], long non-coding RNA and others) has been found to play critical roles in physiological and pathological processes of organisms [38-40], and involved in disease processes including that of cancer. In previous study, we profiled the microRNA expression of nasopharyngeal carcinoma (NPC) and hepatocellular carcinoma (HCC) using a custom microarray

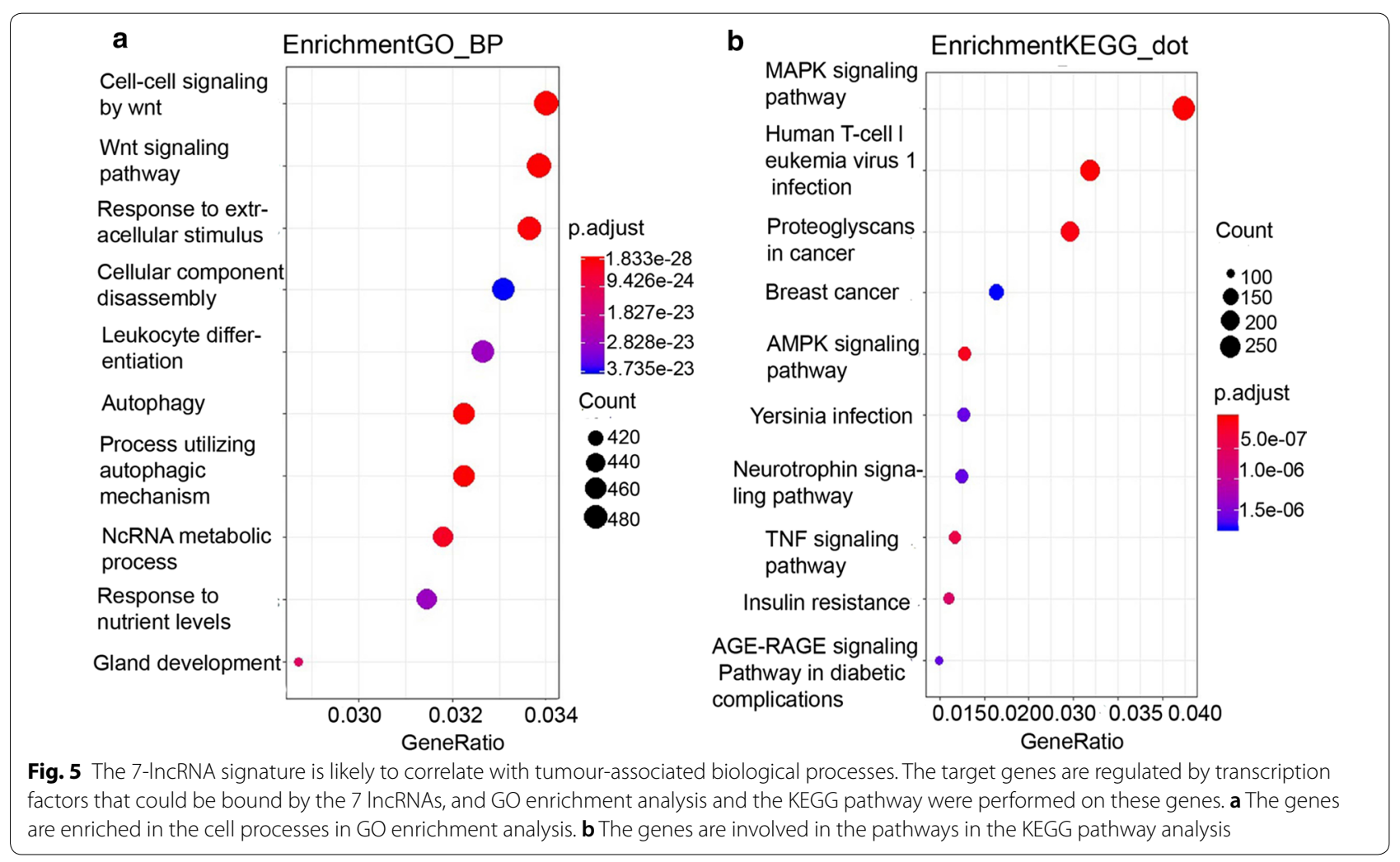


and identified a 5-miRNA signature in NPC [41] and 20-miRNA signature in HCC [26], which were independent prognostic factors and could predict survival and metastasis of patients with NPC and HCC, demonstrating that the two signatures can add more prognostic value to the TNM staging system. In literature reports, lncRNA expression signatures can predict survival in patients with lung adenocarcinoma, colorectal cancer and hepatocellular carcinoma [42-44]. In ESCC patients, although many studies on lncRNA expression profiles have been reported, most studies only employed a few paired cancer and non-cancer samples [45, 46], and only one 3-lncRNA prognostic signature has been identified in 119 esophageal cancers and paired non-cancerous tissues with microarray method [24]. In the present study, we detected 141 ESCC samples with the custom IncRNA microarray and identified a 7-lncRNA signature for predicting survival of patients with ESCC in the training cohort, and validated it with RT-PCR in the independent cohort, suggesting that this 7-lncRNA signature is reliable. We then demonstrated that this signature is an independent prognostic factor with Cox regression analysis and can predict distinct survivals in the patients with the same clinical stage, implying that this signature can provide additional information in predicting survival of ESCC patients. As we expected, our result showed that this 7-IncRNA signature significantly improved the prognostic power of TNM staging system when it combined with TNM stage (Fig. 4), indicating that this signature will be a useful biomarker for predicting survival in ESCC patients. However, this study has limitations: firstly, the custom microarray only includes 2412 lncRNAs, and as a consequence, this signature may not be the best predictor for survival in ESCC; secondly, this study was conducted at a single cancer center and the signature should be further validated in patients from different geographical areas. Nevertheless, our study warrants a multi-center study to further validate the signature's utility and the study of functions and mechanisms of these 7 lncRNAs.

\section{Conclusions}

In this study, we identified a 7-lncRNA signature that can predict the prognosis in patients with ESCC and may help in determining treatment when combined with the TNM stage system.

\section{Supplementary information}

Supplementary information accompanies this paper at https://doi. org/10.1186/s12967-020-02224-z.

Additional file 1. Additional tables.

Additional file 2: Additional figures.

\section{Abbreviations}

ESCC: esophageal squamous cell carcinoma; IncRNAs: long non-coding RNAs; TNM: tumor-node-metastasis; OS: overall survival; DFS: disease-free survival; ROC: receiver operating characteristic; AUC: false discovery rate; QRT-PCR: quantitative real-time reverse transcription polymerase chain reaction; HR: hazard ratio; 95\% Cl: 95\% confidence interval; SAM: significance analysis of microarray; TF: transcription factor; GO: Gene Ontology; KEGG: Kyoto Encyclopedia of Genes and Genomes.

\section{Acknowledgements}

We are grateful to Dr. Qi Wang, PharmD/MD, a resident of UMass Memorial Medical Center \& UMass Medical School, for her proofreading this manuscript.

\section{Authors' contributions}

HYW and JHF conceived and designed this study; GLX provided the clinical specimens; NQW, JC, JW and XZY performed the experiments; MYZ and SJM acquired the data; NQW and JC analyzed the data and results; NQW and JC wrote the manuscript; HYW improved and revised the manuscript. All authors read and approved the final manuscript.

\section{Funding}

This study was supported by Guangdong Esophageal Cancer Institute Science \& Technology program (Grant No: M201412 to HYW) and National Natural

Science Foundation of China (Grant No: 81372564 to HYW).

Availability of data and materials

All data in our study are available upon request.

\section{Ethics approval and consent to participate}

The study was approved by the Research Ethics Committee of Sun Yat-Sen University Cancer Center. Research was conducted according to all ethical standards, and written informed consent was obtained from all patients.

\section{Consent for publication}

Consent to publish has been obtained from all authors.

\section{Competing interests}

The authors declare that they have no competing interests.

\section{Author details}

${ }^{1}$ State Key Laboratory of Oncology in South China, Collaborative Innovation Center for Cancer Medicine, Sun Yat-Sen University Cancer Center, 651 Dongfeng East Road, Building 2, Room 704, Guzngzhou 510060, China. ${ }^{2}$ Guangdong Esophageal Cancer Institute, Guangzhou 510060, China.

${ }^{3}$ Department of Endoscopy and Laser, Sun Yat-Sen University Cancer Center, Guangzhou 510060, China. ${ }^{4}$ Department of Thoracic Oncology, Sun Yat-Sen University Cancer Center, Guangzhou 510060, China. ${ }^{5}$ Department of Oncology, The Second Affiliated Hospital of Nanchang University, Nanchang, China.

Received: 17 September 2019 Accepted: 10 January 2020

Published online: 31 January 2020

References

1. Pennathur A, Gibson MK, Jobe BA, Luketich JD. Oesophageal carcinoma. The Lancet. 2013;381:400-12.

2. Rustgi AK, El-Serag HB. Esophageal carcinoma. N Engl J Med. 2014;371:2499-509.

3. Ohashi S, Miyamoto S, Kikuchi O, Goto T, Amanuma Y, Muto M. Recent advances from basic and clinical studies of esophageal squamous cell carcinoma. Gastroenterology. 2015;149:1700-15.

4. Ishiguro S, Sasazuki S, Inoue M, Kurahashi N, Iwasaki M, Tsugane S, Group JS. Effect of alcohol consumption, cigarette smoking and flushing response on esophageal cancer risk: a population-based cohort study (JPHC study). Cancer Lett. 2009;275:240-6.

5. Varghese TK Jr, Hofstetter WL, Rizk NP, Low DE, Darling GE, Watson TJ, Mitchell JD, Krasna MJ. The society of thoracic surgeons guidelines on the diagnosis and staging of patients with esophageal cancer. Ann Thorac Surg. 2013;96:346-56. 
6. Chung TR, Kim JH, Lee IJ, Cho Y, Kim JW, Lee CG, Jung DH, Park JJ, Youn YH, Park H. Different prognosis of patients with esophageal carcinoma with M1a and regional node involvement. Dig Liver Dis. 2019;51(11):1610-6.

7. Kutup A, Link BC, Schurr PG, Strate T, Kaifi JT, Bubenheim M, Seewald S, Yekebas EF, Soehendra N, Izbicki JR. Quality control of endoscopic ultrasound in preoperative staging of esophageal cancer. Endoscopy. 2007;39:715-9.

8. Kos Z, Dabbs DJ. Biomarker assessment and molecular testing for prognostication in breast cancer. Histopathology. 2016;68:70-85.

9. Hussein MR, Cullen K. Molecular biomarkers in HNSCC: prognostic and therapeutic implications. Expert Rev Anticancer Ther. 2001;1:116-24.

10. Cui XB, Zhang SM, Xu YX, Dang HW, Liu CX, Wang LH, Yang L, Hu JM, Liang WH, Jiang JF, et al. PFN2, a novel marker of unfavorable prognosis, is a potential therapeutic target involved in esophageal squamous cell carcinoma. J Transl Med. 2016;14:137.

11. Deng $H Y$, Wang $Y C, N i P Z$, Lin YD, Chen LQ. Long noncoding RNAs are novel potential prognostic biomarkers for esophageal squamous cell carcinoma: an overview. J Thorac Dis. 2016;8:E653-9.

12. Uraoka N, Oue N, Sakamoto N, Sentani K, Oo HZ, Naito Y, Noguchi T, Yasui W. NRD1, which encodes nardilysin protein, promotes esophageal cancer cell invasion through induction of MMP2 and MMP3 expression. Cancer Sci. 2014;105:134-40

13. Warnecke-Eberz U, Metzger R, Holscher AH, Drebber U, Bollschweiler E. Diagnostic marker signature for esophageal cancer from transcriptome analysis. Tumour Biol. 2016;37:6349-58.

14. Le Bras GF, Farooq MH, Falk GW, Andl CD. Esophageal cancer: the latest on chemoprevention and state of the art therapies. Pharmacol Res. 2016;113:236-44.

15. Chong CR, Janne PA. The quest to overcome resistance to EGFR-targeted therapies in cancer. Nat Med. 2013;19:1389-400.

16. ZhuY, Choi SH, Shah K. Multifunctional receptor-targeting antibodies for cancer therapy. Lancet Oncol. 2015;16:e543-54.

17. Chen M, Huang J, Zhu Z, Zhang J, Li K. Systematic review and meta-analysis of tumor biomarkers in predicting prognosis in esophageal cancer. BMC Cancer. 2013;13:539.

18. Vallbohmer D, Lenz HJ. Predictive and prognostic molecular markers in outcome of esophageal cancer. Dis Esophagus. 2006;19:425-32.

19. Visser E, Franken IA, Brosens LA, Ruurda JP, van Hillegersberg R. Prognostic gene expression profiling in esophageal cancer: a systematic review. Oncotarget. 2017;8:5566-77.

20. Cabianca DS, Casa V, Gabellini D. A novel molecular mechanism in human genetic disease: a DNA repeat-derived IncRNA. RNA Biol. 2012;9:1211-7.

21. Engreitz JM, Haines JE, Perez EM, Munson G, Chen J, Kane M, McDonel PE, Guttman M, Lander ES. Local regulation of gene expression by IncRNA promoters, transcription and splicing. Nature. 2016;539:452-5.

22. Tay Y, Rinn J, Pandolfi PP. The multilayered complexity of ceRNA crosstalk and competition. Nature. 2014;505:344-52.

23. Bhan A, Soleimani M, Mandal SS. Long noncoding RNA and cancer: a new paradigm. Cancer Res. 2017;77:3965-81.

24. Li J, Chen Z, Tian L, Zhou C, He MY, Gao Y, Wang S, Zhou F, Shi S, Feng X, et al. LncRNA profile study reveals a three-IncRNA signature associated with the survival of patients with oesophageal squamous cell carcinoma. Gut. 2014;63:1700-10.

25. Wang HY, Luo M, Tereshchenko IV, Frikker DM, Cui X, Li JY, Hu G, Chu Y, Azaro MA, Lin Y, et al. A genotyping system capable of simultaneously analyzing $>1000$ single nucleotide polymorphisms in a haploid genome. Genome Res. 2005;15:276-83.

26. Wei R, Huang GL, Zhang MY, Li BK, Zhang HZ, Shi M, Chen XQ, Huang $L$, Zhou QM, Jia WH, et al. Clinical significance and prognostic value of microRNA expression signatures in hepatocellular carcinoma. Clin Cancer Res. 2013;19:4780-91.

27. Mao HJ, Li HN, Zhou XM, Zhao JL, Wan DF. Monitoring microarray-based gene expression profile changes in hepatocellular carcinoma. World J Gastroenterol. 2005;11:2811-6.
28. Hu J, He X. Enhanced quantile normalization of microarray data to reduce loss of information in gene expression profiles. Biometrics. 2007;63:50-9.

29. Yu SL, Chen HY, Chang GC, Chen CY, Chen HW, Singh S, Cheng CL, Yu CJ, Lee YC, Chen HS, et al. MicroRNA signature predicts survival and relapse in lung cancer. Cancer Cell. 2008;13:48-57.

30. Pennathur A, Luketich JD. Resection for esophageal cancer: strategies for optimal management. Ann Thorac Surg. 2008;85:S751-6.

31. Rice TW, Blackstone EH, Rusch VW. A cancer staging primer: esophagus and esophagogastric junction. J Thorac Cardiovasc Surg. 2010;139:527-9.

32. Suresh PS, Venkatesh T, Tsutsumi R, Shetty A. Next-generation sequencing for endocrine cancers: recent advances and challenges. Tumour Biol. 2017:39:1010428317698376.

33. Tonella L, Giannoccaro M, Alfieri S, Canevari S, De Cecco L. Gene expression signatures for head and neck cancer patient stratification: are results ready for clinical application? Curr Treat Options Oncol. 2017;18:32.

34. Tang XR, Li YQ, Liang SB, Jiang W, Liu F, Ge WX, Tang LL, Mao YP, He QM, Yang $\mathrm{XJ}$, et al. Development and validation of a gene expression-based signature to predict distant metastasis in locoregionally advanced nasopharyngeal carcinoma: a retrospective, multicentre, cohort study. Lancet Oncol. 2018:19:382-93.

35. Slodkowska EA, Ross JS. MammaPrint 70-gene signature: another milestone in personalized medical care for breast cancer patients. Expert Rev Mol Diagn. 2009;9:417-22.

36. Salazar R, Roepman P, Capella G, Moreno V, Simon I, Dreezen C, LopezDoriga A, Santos C, Marijnen C, Westerga J, et al. Gene expression signature to improve prognosis prediction of stage II and III colorectal cancer. J Clin Oncol. 2011;29:17-24.

37. Stiekema J, Boot H, Aleman BM, Wessels LF, van Sandick JW. Prognostication and prediction using gene expression profiling in oesophageal cancer. Eur J Surg Oncol. 2013;39:17-23.

38. O'Connell RM, Rao DS, Chaudhuri AA, Baltimore D. Physiological and pathological roles for microRNAs in the immune system. Nat Rev Immunol. 2010;10:111-22.

39. Sheng L, Ye L, Zhang D, Cawthorn WP, Xu B. New insights into the long non-coding RNA SRA: physiological functions and mechanisms of action. Front Med (Lausanne). 2018:5:244.

40. Calore F, Lovat F, Garofalo M. Non-coding RNAs and cancer. Int J Mol Sci. 2013;14:17085-110.

41. Liu N, Chen N-Y, Cui R-X, Li W-F, Li Y, Wei R-R, Zhang M-Y, Sun Y, Huang $\mathrm{B}-\mathrm{J}$, Chen $\mathrm{M}$, et al. Prognostic value of a microRNA signature in nasopharyngeal carcinoma: a microRNA expression analysis. Lancet Oncol. 2012;13:633-41.

42. Chen M, Liu B, Xiao J, Yang Y, Zhang Y. A novel seven-long non-coding RNA signature predicts survival in early stage lung adenocarcinoma. Oncotarget. 2017;8:14876-86.

43. Fan Q, Liu B. Discovery of a novel six-long non-coding RNA signature predicting survival of colorectal cancer patients. J Cell Biochem. 2018;119:3574-85.

44. Gu JX, Zhang X, Miao RC, Xiang XH, Fu YN, Zhang JY, Liu C, Qu K. Six-long non-coding RNA signature predicts recurrence-free survival in hepatocellular carcinoma. World I Gastroenterol. 2019;25:220-32.

45. Yao J, Huang JX, Lin M, Wu ZD, Yu H, Wang PC, Ye J, Chen P, Wu J, Zhao GJ. Microarray expression profile analysis of aberrant long non-coding RNAs in esophageal squamous cell carcinoma. Int J Oncol. 2016;48:2543-57.

46. Wang J, Sun D, Wu K, Liu J, Zhao M, Li X, Xu Y, Li B. Genome-wide analysis of long non-coding RNAs in esophageal squamous cell carcinoma reveals their potential role in invasion and metastasis. Thorac Cancer. 2019:10:78-89.

\section{Publisher's Note}

Springer Nature remains neutral with regard to jurisdictional claims in published maps and institutional affiliations. 